\title{
SOLAR NEUTRINOS AS BACKGROUND IN DIRECT DARK MATTER SEARCHES.
}

\author{
J. D. Vergados*, H. Ejiri ${ }^{\dagger}$ and I. Giomataris** \\ * Physics Department, University of Ioannina, Ioannina, GR 451 10, Greece and \\ Theory Division, CERN 1211, Geneva 23, CH. \\ ${ }^{\dagger} R C N P$, Osaka University, Osaka, 567-0047, Japan and \\ National Institute of Radiological Sciences, Chiba, 263-8555, Japan. \\ ** IRFU, Centre d' etudes de Saclay, 91191 Gif sur Yvette CEDEX, France.
}

\begin{abstract}
The coherent contribution of all neutrons in neutrino nucleus scattering due to the neutral current is examined considering the boron solar neutrinos. These neutrinos could potentially become a source of background in the future dark matter searches aiming at nucleon cross sections in the region well below the $10^{-10} \mathrm{pb}$, i.e a few events per ton per year.
\end{abstract}

Keywords: WIMPs, LSP, Dark Matter, Direct detection, Boron neutrinos, Quenching factor, Differential rates, Total rates, Neutral current, Neutron coherence

PACS: $13.15 .+\mathrm{g}, 14.60 \mathrm{Lm}, 14.60 \mathrm{~Bq}, 23.40 .-\mathrm{s}, 95.55 . \mathrm{Vj}, 12.15 .-\mathrm{y}$.

\section{INTRODUCTION.}

The universe is observed to contain large amounts of dark matter [1, 2], and its contribution to the total energy density is estimated to be $\sim 25 \%$. Even though there exists firm indirect evidence from the halos of dark matter in galaxies and clusters of galaxies it is essential to detect matter directly.

The possibility of direct detection, however, depends on the nature of the dark matter constituents, i.e the WIMPs (weakly interacting massive particles). Supersymmetry naturally provides candidates for these constituents [3]. In the most favored scenario of supersymmetry, the lightest supersymmetric particle (LSP) can be described as a Majorana fermion, a linear combination of the neutral components of the gauginos and higgsinos. Other possibilities also exist, see, e.g. some models in universal theories with extra dimensions [4],[5]. Since the WIMPs are expected to be very massive $\left(m_{W I M P} \geq\right.$ $30 \mathrm{GeV}$ ) and extremely non-relativistic with average kinetic energy $\langle T\rangle \simeq 50 \mathrm{keV}$ $\left(m_{W I M P} / 100 \mathrm{GeV}\right)$, a WIMP interaction with a nucleus in an underground detector is not likely to produce excitation. As a result, WIMPs can be directly detected mainly via the recoil of a nucleus $(A, Z)$ in elastic scattering. The event rate for such a process can be computed by a standard procedure (see recent work [6] and references therein). Since the particle physics parameters will most likely result in very small cross sections, the future dark matter experiments, like the XENON-ZEPLIN [7], aim at detecting 10 events per ton per year. At this level one may encounter very bothersome backgrounds. One such background may come from the high energy boron solar neutrinos (the other neutrinos are characterized either by too small energy or much lower fluxes).

During the last years various detectors aiming at detecting recoiling nuclei have been 
developed in connection with dark matter searches [7] with thresholds in the few keV region. Furthermore it has become feasible to detect neutrinos by measuring the recoiling nucleus in gaseous detectors with much lower threshold energies [8], explpoiting the neutral current interzction [9]. This interaction, through its vector component, can lead to coherence, i.e. an additive contribution of all neutrons in the nucleus.

In this paper we will calculate the recoil spectrum for boron solar neutrinos and compare it with that associated with WIMPs, both for a light and a heavy target.

\section{A BRIEF DISCUSSION OF THE RATES FOR DIRECT WIMP DETECTION}

Before proceeding with the evaluation of the event rate for nuclear recoils originating from the neutrino nuclear scattering we will briefly summarize the results recently obtained [6]. The event rate for the coherent WIMP-nucleus elastic scattering, which is given by:

$$
\begin{aligned}
R= & \frac{\rho(0)}{m_{\chi^{0}}} \frac{m}{m_{p}} \sqrt{\left\langle v^{2}\right\rangle} \sigma_{p, \chi^{0}}^{S} f_{c o h}\left(A, \mu_{r}(A)\right) \\
& f_{c o h}\left(A, \mu_{r}(A)\right)=\frac{100 \mathrm{GeV}}{m_{\chi^{0}}}\left[\frac{\mu_{r}(A)}{\mu_{r}(p)}\right]^{2} A t_{c o h}\left(1+h_{c o h} \cos \alpha\right)
\end{aligned}
$$

where $\sigma_{p, \chi^{0}}^{S}$ is the coherent WIMP-nucleon cross section, $\mu_{r}(A)$ the WIMP-nucleus reduced mass The parameter $t_{c o h}$ takes into account the folding of the nuclear form factor with the WIMP velocity distribution, $h_{c o h}$ deals with the modulation [10] due to the motion of the Earth and $\alpha$ is the phase of the Earth (zero around June 2nd).

The number of events in time $t$ due to the scalar interaction, which leads to coherence [6], is:

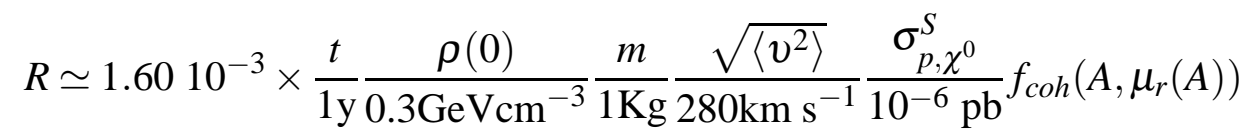

Assuming a constant nucleon cross section we get the differential rate indicated in Fig. 1. The total (time averaged) coherent event rate is shown in Fig. 2.
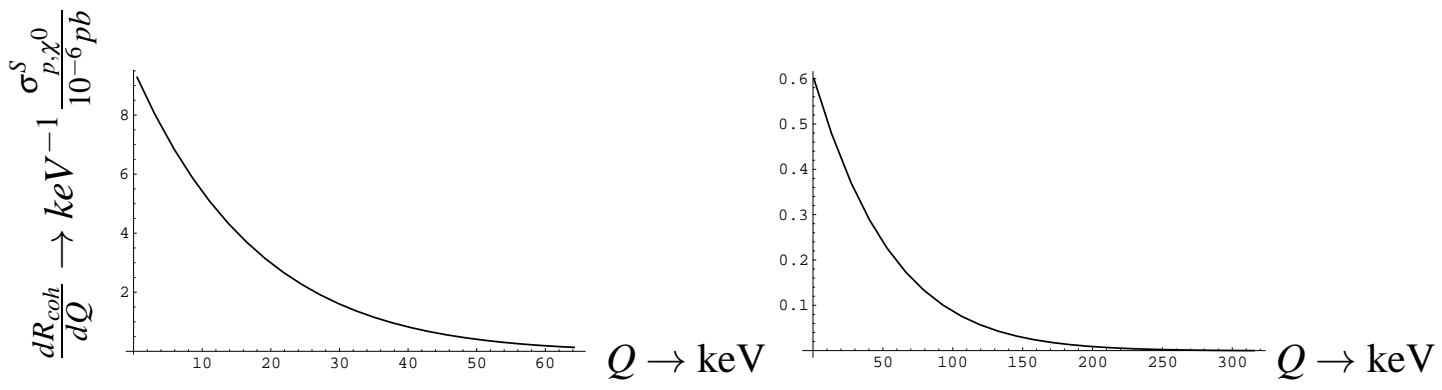

FIGURE 1. We show the differential event rate $d R_{c o h} / d Q$ for the coherent process, as a function of the energy transfer, for a WIMP mass, $m_{\chi}$, of $100 \mathrm{GeV}$ in the case of ${ }^{131} \mathrm{Xe}$ on the left and ${ }^{32} \mathrm{~S}$ on the right. 

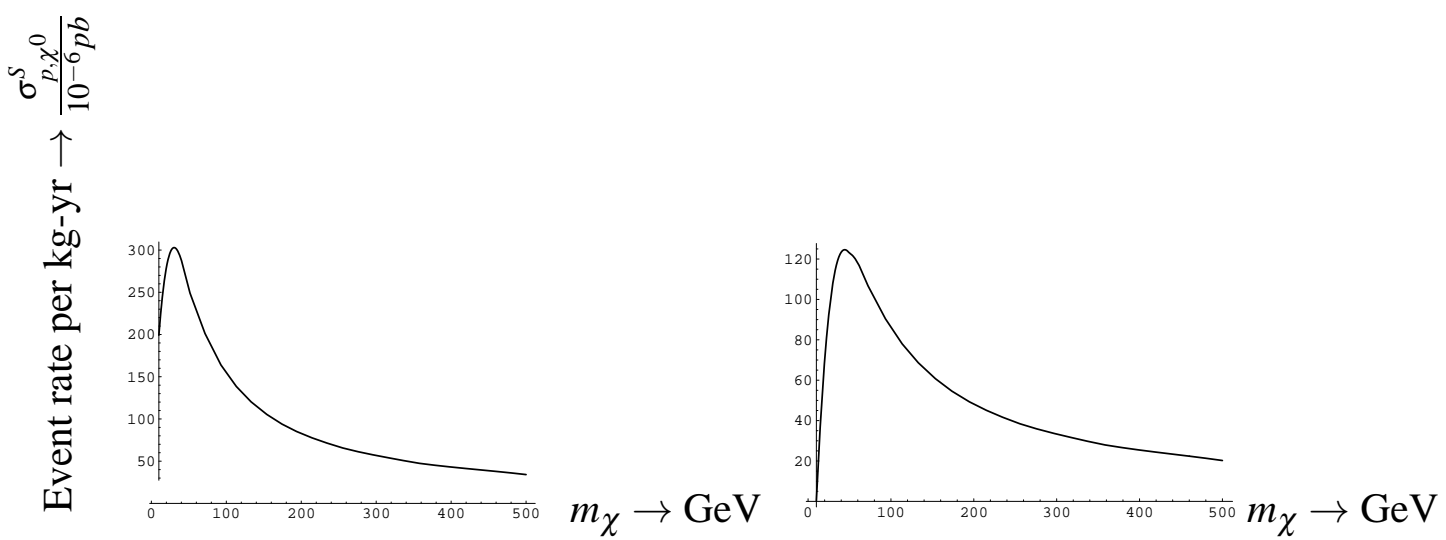

FIGURE 2. We show the total event rate for the coherent process as a function of the WIMP mass in the case of ${ }^{131} \mathrm{Xe}$ for zero threshold on the left ans $10 \mathrm{keV}$ on the right.

\section{ELASTIC NEUTRINO NUCLEON SCATTERING}

The cross section for elastic neutrino nucleon scattering has extensively been studied. It has been shown that at low energies it can be simplified and be cast in the form: [11],[12]:

$$
\left(\frac{d \sigma}{d T_{N}}\right)_{w e a k}=\frac{G_{F}^{2} m_{N}}{2 \pi}\left[\left(g_{V}+g_{A}\right)^{2}+\left(g_{V}-g_{A}\right)^{2}\left[1-\frac{T_{N}}{E_{V}}\right]^{2}+\left(g_{A}^{2}-g_{V}^{2}\right) \frac{m_{N} T_{N}}{E_{V}^{2}}\right]
$$

where $T_{n}$ is the energy of the nucleon, $m_{N}$ the nucleon mass and $g_{V}, g_{A}$ are the weak coupling constants:

$g_{V}=-2 \sin ^{2} \theta_{W}+1 / 2 \approx 0.04, g_{A}=\frac{1.27}{2},(v, p) ; g_{V}=-1 / 2, g_{A}=-\frac{1.27}{2},(v, n)$

The elastic neutrino nucleus scattering is given by:

$$
\left(\frac{d \sigma}{d T_{A}}\right)_{\text {weak }}=\frac{G_{F}^{2} A m_{N}}{2 \pi}\left(N^{2} / 4\right) F^{2}\left(q^{2}\right)\left(1+\left(1-\frac{T_{A}}{E_{V}}\right)^{2}-\frac{A m_{N} T_{A}}{E_{V}^{2}}\right)
$$

with $T_{A}$ is the energy of the recoiling nucleus and $F\left(q^{2}\right)=F\left(T_{A}^{2}+2 A m_{N} T_{A}\right)$

To proceed further we must convolute the cross section with the neutrino spectrum. For the relwvant boron solar neutrinos the normalized spectrum is shown in Fig. 3 , The expected total neutrino flux is $\Phi_{v}=5.15 \times 10^{6} \mathrm{~cm}^{2} \mathrm{~s}-1$. The obtained differential cross section is shown in Fig. 4. Note that the cross section has been almost completely depleted beyond energies 1 and $5 \mathrm{keV}$ for a intermediate $\left({ }^{131} \mathrm{Xe}\right)$ and light $\left({ }^{32} \mathrm{~S}\right)$ targets respectively. Integrating the differential cross section down to zero threshold we find the event rates given in table 1 . The event rates are almost two orders of magnitude smaller than the rates for WIMP detection obtained with a nucleon cross section of $10^{-9} \mathrm{pb}$. Thus such neutrinos cannot be a serious background for WIMP searches in the region $10^{-9}-10^{-10} \mathrm{pb}$. In any event, as we will see below, the neutrino induced recoils are less of a background problem in the realistic case of non zero energy threshold. 


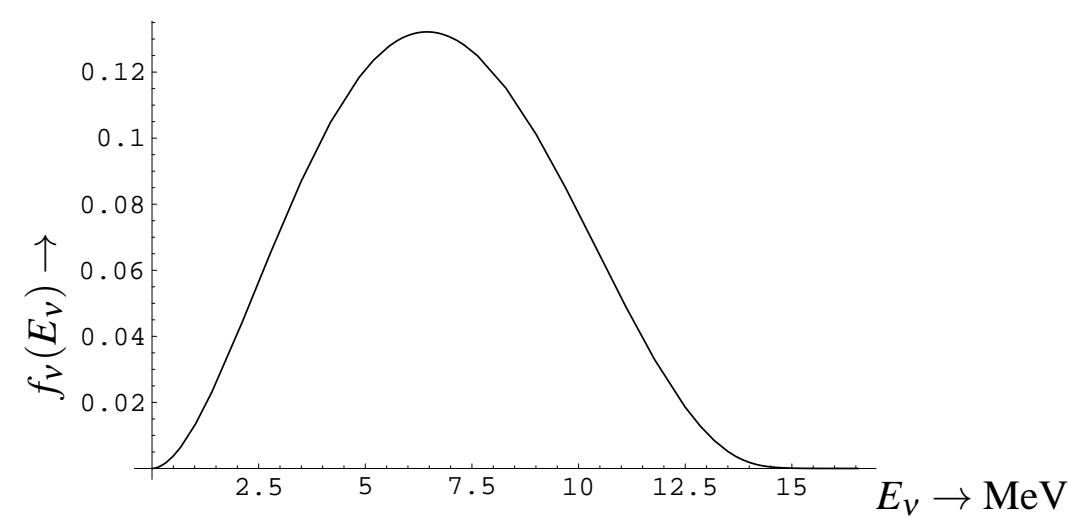

FIGURE 3. The boron solar neutrino spectrum. The total neutrino flux is $\Phi_{v}=5.15 \times 10^{6} \mathrm{~cm}^{2} \mathrm{~s}^{-1}$.
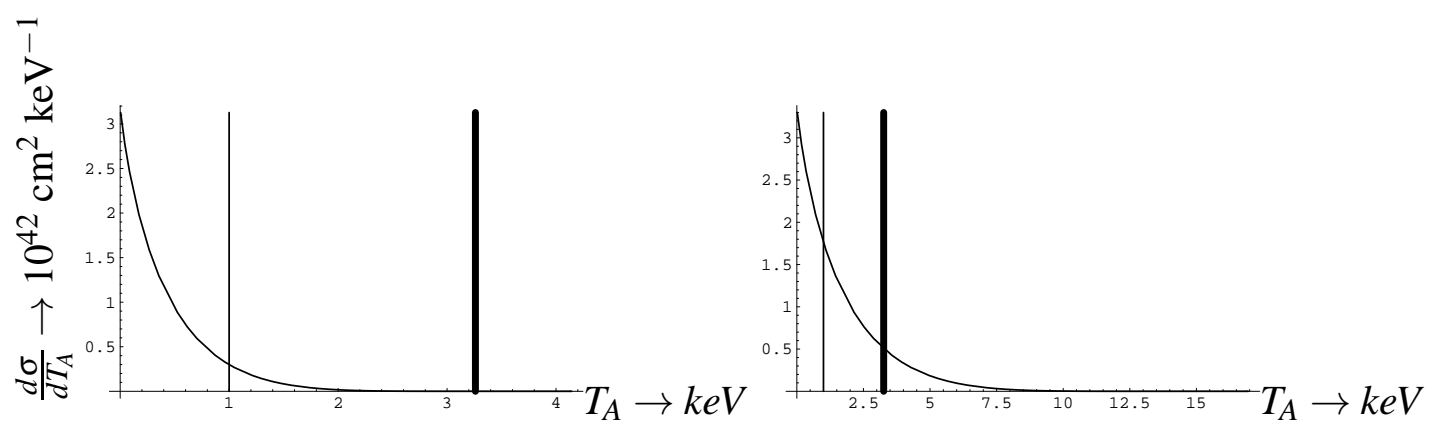

FIGURE 4. The neutrino induced differential cross section in units $10^{-42} \mathrm{~cm}^{2} \mathrm{keV}^{-1}$ as a function of the recoil energy in $\mathrm{keV}$ in the case of the target ${ }^{131} \mathrm{Xe}$ on the left and ${ }^{32} \mathrm{~S}$ on the right. Note that essentially all the contribution comes from the recoil energy region $0 \leq T_{A} \leq 1 \mathrm{keV}$. The available kinematic region for a threshold energy of $1 \mathrm{keV}$ is that which is above the thin line (thick line) for no quenching (quenching) respectively. The effect of the form factor is invisible in the figure.

We should mention that the obtained rates are independent of the neutrino oscillation parameters, since the neutral current events are not affected by such oscillations. The above results shown in table 1), refer to an ideal detector with zero energy threshold. For a real detector, however, the nuclear recoil events are quenched, especially at low energies. The quenching factor for a given detector is the ratio of the signal height for a recoil track divided by that of an electron signal height with the same energy. The actual quenching factors must be determined experimentally for each target. In the present work we considered [6] the phenomenological factor :

$$
Q_{f a c}\left(T_{A}\right)=r_{1}\left[\frac{T_{A}}{1 k e V}\right]^{r_{2}}, r_{1} \simeq 0.256, r_{2} \simeq 0.153
$$

Due to the relatively low recoil energies the effect of threshold is crucial (see Fig 5). One clearly sees that the observed events are an order of magnitude down if the energy threshold is $1 \mathrm{keV}(2 \mathrm{keV})$ for $\mathrm{Xe}(\mathrm{S})$ respectively. Thus with quenching most signals get below the threshold energy of $\approx 1 \mathrm{keV}$. On the other hand the WIMP event rates are almost unaffected, unless the threshold energy becomes larger than $5 \mathrm{keV}$. 
TABLE 1. Comparison of the event rates for boron solar neutrino and WIMP detection. In evaluating the latter we assumed a nucleon cross section independent of the mass. The kinematics were obtained assuming two WIMP masses, namely 100 and $300 \mathrm{GeV}$. NoFF means that the nuclear form factor was neglected.

\begin{tabular}{|c|c|c|c|c|}
\hline target & $R_{\chi}(\mathrm{kg}-\mathrm{y}) \times \frac{\sigma_{N}}{10^{-9} p b}$ & $R_{\chi}(\mathrm{kg}-\mathrm{y}) \times \frac{\sigma_{N}}{10^{-9} p b}$ & $R_{v}(\mathrm{~kg}-\mathrm{y})$ & $R_{V}(\mathrm{~kg}-\mathrm{y}) ;$ NoFF \\
\hline & $m_{\chi}=100 \mathrm{GeV}$ & $m_{\chi}=300 \mathrm{GeV}$ & & \\
\hline${ }^{131} \mathrm{Xe}$ & 0.167 & 0.060 & $0.934 \times 10^{-3}$ & $0.952 \times 10^{-3}$ \\
${ }^{32} \mathrm{~S}$ & 0.033 & 0.014 & $0.167 \times 10^{-3}$ & $0.168 \times 10^{-3}$ \\
\hline
\end{tabular}
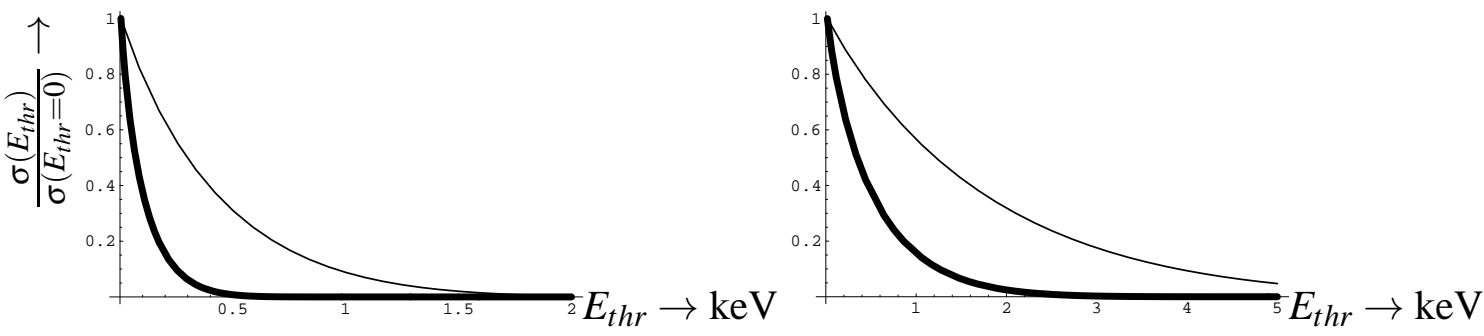

FIGURE 5. The ratio of the total cross section with threshold divided by that with zero threshold respectively. The thick (thin) line correspond to quenching (no quenching). Note that the observed events are an order of magnitude down, if the energy threshold is $1 \mathrm{keV}$.

\section{CONCLUDING REMARKS}

In the present study we considered the elastic scattering of WIMP-nucleus interaction and the corresponding elastic scattering of boron solar neutrinos. Our results can be summarized as follows:

1. The differential cross section for solar neutrinos decreases sharply as the nuclear recoil energy increases. It almost vanishes beyond $1 \mathrm{keV}(5 \mathrm{keV})$ for intermediate (light target), like ${ }^{131} \mathrm{Xe}\left({ }^{32} \mathrm{~S}\right)$. On the other the corresponding event rates for WIMPs of mass $\approx 100 \mathrm{GeV}$ extend further than $30(150) \mathrm{keV}{ }^{131} \mathrm{Xe}\left({ }^{32} \mathrm{~S}\right)$ respectively.

2. The event rates for boron solar neutrinos at zero threshold energy and no quenching are 2-3 orders of magnitude smaller than those for WIMPs with a nucleon cross section $10^{-9} \mathrm{pb}$. Thus solar neutrinos are not a serious background down to $10^{-10} \mathrm{pb}$.

3. Since the nuclear recoil energy in solar neutrino scattering is smaller than that associated with heavy WIMPs, one can further substantially decrease its contribution by restricting the observation above a few $\mathrm{keV}$ without seriously affecting the corresponding WIMP rates. Thus neutrinos do not appear to be a serious background even at the level of $10^{-11} \mathrm{pb}$.

4. One may reduce this background still further by exploiting the quenching factors.

5. One may diminish this bakground further by exploiting the annual modulation of the signal (see e.g. [10] and references there in) or even better by performing directional experiments [13],[14], i.e experiments in which the direction of recoil 
is also measured, one will be able to select WIMP signals and discriminate against neutrino scattering.

\section{ACKNOWLEDGMENTS}

One of the authors (JDV) is indebted to MRTN-CT-2004-503369 for support and financing his participation in DSU2008. Special thanks to BUE and professor S. Khalil for their hospitality as well as to the CERN Theory Division for support and hospitality.

\section{REFERENCES}

1. D.N. Spergel et al, Three-Year WMAP Results: Implications for Cosmology, astro-ph/0603449;

L. Page et al, Three-Year WMAP Results: Polarization Analysis, astro-ph/0603450,

G. Hinsaw et al, Three-Year WMAP Observations: Implications Temperature Analysis, astro-ph/0603451,

N Jarosik et al, Three-Year WMAP Observations: Beam Profiles, Data Processing, Radiometer Characterization and Systematic Error Limits, astro-ph/0603452.

2. D.N. Spergel et al, Astrophys. J. Suppl. 148, 175 (2003).

3. M. W. Goodman and E. Witten, Phys. Rev. D 31, 3059 (1985); T. S. Kosmas and J. D. Vergados, Phys. Rev. D 55, 1752 (1997); J. Ellis and L. Roszkowski, Phys. Lett. B 283, 252 (1992); A. Bottino et al., Phys. Lett B 402, 113 (1997);

R. Arnowitt. and P. Nath, Phys. Rev. Lett. 74, 4592 (1995); Phys. Rev. D 54, 2374 (1996); hep-ph/9902237;

V. A. Bednyakov, H.V. Klapdor-Kleingrothaus and S.G. Kovalenko, Phys. Lett. B 329, 5 (1994); Update on the Direct Detection of Supersymmetric Dark Matter J. Ellis, K. A. Olive, Y. Santoso, V. C. Spanos, hep-ph/0502001.

4. See, e.g., G. Servant, in Les Houches :Physics at TeV Colliders 2005" Beyond the Standard Model working group: summary report, B.C. Allanach (ed.), C. Grojean (ed.), P. Skands (ed.), al, section 25, p.164; hep-ph/0602198.

5. V. Oikonomou, J. Vergados, and C. C. Moustakidis, Nuc. Phys. B 773, 19 (2007).

6. J.D. Vergados and H. Ejiri, Nuc. Phys., in press arXiv:0805.2583.

7. See, e.g,

R. Bernabei et al., it Phys. Lett. B 389 (1996) 757 . R. Bernabei et al., Phys. Lett. B 424 (1998) 195; B 450448 (1999) 448.

A. Benoit et al, [EDELWEISS collaboration], Phys. Lett. B 54543 (2002) 43; V. Sanglard et al [EDELWEISS collaboration], Phys. Rev. D 71 (2005) 122002.

D.S. Akerib et al, [CDMS Collaboration], Phys. Rev D 68 (2003) $082002 \sqrt{\operatorname{arXiv}: a s t r o-p h / 0405033}$

G. Alner et al, (UK Dark Matter Collaboration), Astropar. Physics 23 (2005) 444;

G. J. Alner et al, ZEPLIN II Collaboration, arXiv:astro-ph/0701858, D. Yu. Akimov et al, ZEPLIN

II Collaboration, Astropart.Phys. 27 (2007) 46; H. M. Araujo et al, ZEPLIN II Collaboration, Astropart.Phys. 26 (2006) 140-153.

TA Girard et al, The SIMPLE Collaboration, Phys.Lett. B 621 (2005) 233; arXiv:hep-ex/0505053.

8. Y. Giomataris and J. Vergados, Phys. Lett. B 634, 23 (2006), ; hep-ex/0503029.

9. A. Burrows, D. Klein, and R. Gandhi, Phys. Rev. D 45, 3361 (1992).

10. J. D. Vergados, Phys. Rev. D 57, 103003 (2003), hep-ph/0303231.

11. J. Beacom, W. Farr, and P. Vogel, Phys. Rev. D 66, 03301 (2002).

12. P. Vogel and J. Engel, Phys. Rev. D 39, 3378 (1989).

13. The NAIAD experiment B. Ahmed et al, Astropart. Phys. 19 (2003) 691; hep-ex/0301039

B. Morgan, A. M. Green and N. J. C. Spooner, Phys. Rev. D 71 (2005) 103507; astro-ph/0408047.

14. J. Vergados and A. Faessler, Phys. Rev. D 75, 055007 (2007). 